\title{
Characteristic and Synthetic Approach of Molecularly Imprinted Polymer
}

\author{
Hongyuan Yan and Kyung Ho Row* \\ Center for Advanced Bioseparation Technology, Department of Chemical Engineering, Inha University, \\ 253 Yonghyun-Dong, Nam-Ku, Incheon, 402-751, Korea \\ * Corresponding author. E-mail: rowkho@inha.ac.kr
}

Received: 12 April 2006 / Accepted: 27 June 2006 / Published: 29 June 2006

\begin{abstract}
Molecularly imprinted polymers (MIP) exhibiting high selectivity and affinity to the predetermined molecule (template) are now seeing a fast growing research. However, optimization of the imprinted products is difficult due to the fact that there are many variables to consider, some or all of which can potentially impact upon the chemical, morphological and molecular recognition properties of the imprinted materials. This review present a summary of the principal synthetic considerations pertaining to good practice in the polymerization aspects of molecular imprinting, and is primarily aimed at researcher familiar with molecular imprinting methods but with little or no prior experience in polymer synthesis. The synthesis, characteristic, effect of molecular recognition and different preparation methods of MIP in recent few years are discussed in this review, unsolved problems and possible developments of MIP were also been briefly discussed.
\end{abstract}

Keywords: molecularly imprinted polymer, special molecular recognition, synthetic approach

\section{Molecular Imprinting Technology}

Molecular imprinting technology is a rapidly developing technique for the preparation of polymers having specific molecular recognition properties for a given compound, its analogues or for a single enantiomer [1-3]. Synthesis of MIP is a relatively straightforward and inexpensive procedure. In short, the molecularly imprinted polymer is prepared by mixing the template molecule with functional monomers, cross-linking monomers and a radical initiator in a proper solvent, most often an aprotic and non polar solvent. Subsequently, this pre-polymerization mixture is irradiated with UV light or 
subjected to heat in order to initiate polymerization. During polymerization, the complexes formed between the template molecule and the functional monomers will be stabilized within the resulting rigid, highly cross-linked polymer. After polymerization and extracted out of the template molecule, the resulting imprinted polymer possessing a permanent memory for the imprint species are formed, enabling the resultant polymer selectively to rebind the imprint molecule from a mixture of closely related compounds. The three-dimensional cavities that are complementary in both shape and chemical functionality arrangement to those of the template be left in the polymer matrix and the high degree of cross-linking enables the microcavities to maintain their shape after removal of the template, and thus, the functional groups are held in an optimal configuration for rebinding the template, allowing the receptor to 'recognize' the original substrate [4-5]. Molecularly imprinted polymers demonstrate very good thermal and chemical stability and can be used in aggressive media [6]. MIP possess several advantages over their biological counterparts including low cost, ease of preparation, storage stability, repeated operations without loss of activity, high mechanical strength, durability to heat and pressure, and applicability in harsh chemical media. As a technique for the creation of artificial receptor-like binding sites with a 'memory' for the shape and functional group positions of the template molecule, molecular imprinting has become increasingly attractive in many fields of chemistry and biology, particularly as an affinity material for sensors [7-11], binding assays [12], artificial antibodies [13-14], adsorbents for solid phase extraction [15-19], and chromatographic stationary phases [20-23].

\section{Category of MIP}

Essentially, two kinds of molecular imprinting strategies have been established based on covalent bonds or non-covalent interactions between the template and functional monomers (Figure 1). In both cases, the functional monomers, chosen so as to allow interactions with the functional groups of the imprinted molecule, are polymerized in the presence of the imprinted molecule. The special binding sites are formed by covalent or, more commonly, non-covalent interaction between the functional group of imprint template and the monomer, followed by a crosslinked co-polymerization [24]. Of the two strategies, the non-covalent approach has been used more extensively due to follow three reasons:

(1) Non-covalent protocol is easily conducted, avoiding the tedious synthesis of prepolymerization complex.

(2) Removal of the template is generally much easier, usually accomplished by continuous extraction.

(3) A greater variety of functionality can be introduced into the MIP binding site using non-covalent methods. 


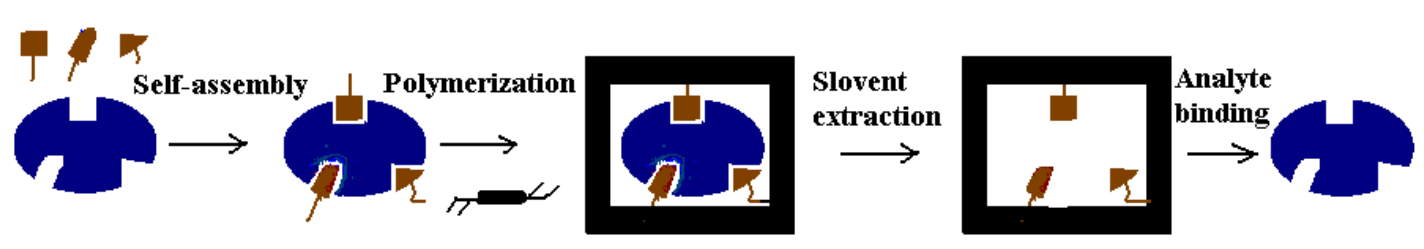

Noncovalent imprinting

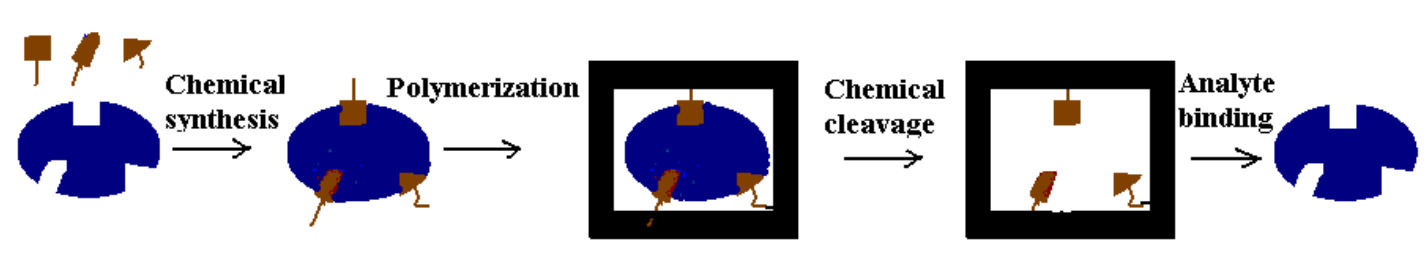

Covalent imprinting

Figure 1. Schematic representation of covalent and non-covalent molecular imprinting procedures

\subsection{Covalent Approach}

In covalent approach, the imprinted molecule is covalently coupled to a polymerizable molecule. The binding of this type of polymer-relies on reversible covalent bonds. After copolymerization with crosslinker, the imprint molecule is chemically cleaved from the highly crosslinked polymer. Wulff [25-26] and co-workers first produced MIP by synthesizing specific sugar or amino acid derivatives which contained a polymerisable function such as vinylphenylboronate by covalent imprinting methods. After polymerization they hydrolyzed the sugar moiety and used the polymer for selective binding and result shown that for covalent molecular imprinting, selectivity of MIP increases with maximization of crosslinker. Moreover, the requirements of covalent imprinting are different than those for non-covalent imprinting, particularly with respect to ratios of functional monomer, crosslinker, and template. However, since the choice of reversible covalent interactions and the number of potential templates are substantially limited, reversible covalent interactions with polymerizable monomers are fewer in number and often require an acid hydrolysis procedure to cleave the covalent bonds between the template and the functional monomer.

\subsection{Non-covalent Approach}

Non-covalent approach is the most frequently used method to prepare MIP due to its simplicity. During the non-covalent approach, the special binding sites are formed by the self-assembly between the template and monomer, followed by a crosslinked co-polymerization [27-28]. The imprint molecules interact, during both the imprinting procedure and the rebinding, with the polymer via non-covalent interactions, e.g. ionic, hydrophobic and hydrogen bonding. The non-covalent imprinting approach seems to hold more potential for the future of molecular imprinting due to the vast number of compounds, including biological compounds, which are capable of non-covalent interactions with functional monomers [29-30]. Limits to the non-covalent molecular imprinting are set by the peculiar molecular recognition conditions. Most of fact, the formation of interactions between monomers and 
the template are stabilized under hydrophobic environments, while polar environments disrupt them easily. Another limit is represented by the need of several distinct points of interactions: some molecules characterized by a single interacting group, such as an isolated carboxyl, generally give imprinted polymers with very limited molecular recognition properties, which have little interest in practical applications.

Understanding the basic optimization of non-covalent methods is important for two reasons: the methodology is far easier than covalent methods, and it produces higher affinity binding sites, versus covalent methods. The trends in binding and selectivity in non-covalently imprinted polymers are explained best by incorporates multiple functional monomers for the highest affinity binding sites. The increased number of binding interactions in the polymer binding site may account for greater fidelity of the site, and thus impart greater affinity and selectivity to the site. This would suggest that the number of functional groups in the polymer binding site is not determined directly by the solution phase pre-polymer complex; rather, it is determined during polymerization. Because of the difficulty to characterizing the binding site structures during and after polymerization, the actual events determining the final binding site structure are still a main challenge.

\section{Molecular Recognition of MIP}

Despite the wealth of literature on molecular imprinting technology that has been published within past decades, the mechanisms of recognition and their rational control appear not entirely understood, thus inhibiting optimization of the imprinting strategy. Molecular recognition ability is dependent on several factors, such as shape complementarity, functional complementarity, contributions from the surrounding environment. As for the functional complementarity, even though all non-covalent interactions are applicable to the molecular recognition between a target molecule and a molecular recognition site formed by a molecular imprinting, the nature of the template, monomers and the polymerization reaction itself determine the quality and performance of the polymer product. Moreover, the quantity and quality of the molecularly imprinted polymer recognition sites is a direct function of the mechanisms and extent the monomer-template interactions present in the pre-polymerization mixture. The recognition of the polymer constitutes an induced molecular memory, which makes the recognition sites capable of selectively recognizing the imprint species.

The imprinted molecules interact, during both the imprinting procedure and the rebinding, with the polymer via non-covalent interactions, e.g. ionic, hydrophobic and hydrogen bonding [31]. Hydrogen bond is most often applied as a molecular recognition interaction of molecularly imprinted polymers. From this, acrylic acid and methacrylic acid have usually been adopted as functional monomers since carboxyl group functions as a hydrogen donor and a hydrogen acceptor at the same time. These non-covalent interactions are easily reversed, usually by a wash in aqueous solution of an acid, a base, or methanol, thus facilitating the removal of the template molecule from the network after polymerization. In addition to the better versatility of this more general approach, it allows fast and reversible binding of the template. 
Mosbach et al. [32-34] has shown various examples of MIP prepared from different functional monomers (e.g. methacrylic acid (MAA) and acrylamide (AM)) and a cross-linking copolymer (e.g. ethyleneglycol dimethacrylate (EDMA)) in porogenic solvents such as chloroform, toluene, tetrahydrofuran and acetonitrile. Most MIP are prepared by non-covalent imprinting and the common systems are based on methacrylic monomers, such as methacrylic acid because its carboxyl group is the most common hydrogen-bonding and acidic functional group in molecular imprinting, cross-linked with EDMA. Molecularly imprinted polymers prepared with the trifunctional crosslinkers pentaerythritol triacrylate and trimethlolpropane trimethacrylate (TRIM) were shown to be superior to those prepared with EDMA, in that higher load capacities and better resolution were obtained.

Nicholls [35-36] studied thermodynamic considerations of MIP recognition. He explained that the extent of template complexation at equilibrium is governed by the change in Gibbs free energy of formation of each mode of template-functional monomer interaction. As the prearrangement phase is under thermodynamic control, the monomer(s)-template complex is not subjected to conformational strains and unfavourable vander Waals interactions. Furthermore, MIP only undergoes limited changes in its conformation during the recognition of the template because of its high degree of cross-linking. Since both polymerization and rebinding processes occurred generally in lipophylic solvents, hydrophobic interactions can be considered negligible.

Kim et al. [37] investigated and compared the thermodynamic properties of copolymers imprinted for Fmoc-L-tryptophan and prepared by two different methods: in situ polymerization and traditional bulk method. The thermodynamic properties of the two different MIP showed that three types of binding sites coexist on their surface. The highest energy sites adsorb only the imprinted molecule or template. Most of the intermediate energy sites adsorb both the template and its antipode, although part of them may adsorb only the template. Finally, the lowest energy sites provide nonselective interactions of both the template and its antipode. On the nonimprinted copolymer, there are only two types of sites. The high-energy sites have a slightly lower energy that the intermediate sites of the MIP and the low-energy sites have properties close to those of the lowest energy sites on the MIP. The monolithic MIP has fewer nonselective sites than the bulk MIP.

A layer of mineral oil was deposited onto the surface of the polymer in order to create a hydrophobic environment in the binding sites and to improve the recognition properties of the polymer in polar solvents was investigated by Piletska [38]. The performances of polymers in acetonitrile showed that the modified polymers possessed significantly increased selectivity as compared with non-treated ones. The three-fold improvement of recognition ability to template (cocaine) was achieved; at the same time, for non-specific molecule (morphine) the improvement was only 1.3 times. The investigation of the stability of mineral oil coating on the polymer surface suggested that the effect produced is stable over a long period of time. This approach could be used to broaden the range of experimental conditions where molecularly imprinted polymers can perform successfully. 


\section{Effecting of Special Molecular Recognition}

\subsection{Optimization of the Polymer Structure}

The synthesis of molecularly imprinted polymers is a chemically complex pursuit and demands a good understanding of chemical equilibrium, molecular recognition theory, thermodynamics and polymer chemistry in order to ensure a high level of molecular recognition [39-43]. The polymers should be rather rigid to preserve the structure of the cavity after splitting off the template. On the other hand, a high flexibility of the polymers should be present to facilitate a fast equilibrium between release and reuptake of the template in the cavity. These two properties are contradictory to each other, and a careful optimization became necessary. The challenge of designing and synthesizing a molecularly imprinted polymer can be a daunting prospect to the uninitiated practitioner, not least because of the sheer number of experimental variables involved, e.g. the nature and levels of template, functional monomer(s), cross-linker(s), solvent(s) and initiator, the method of initiation and the duration of polymerization. Moreover, optimization of the imprinted products is made more difficult due to the fact that there are many variables to consider, some or all of which can potentially impact upon the chemical, morphological and molecular recognition properties of the imprinted materials. Fortunately, in some instances it is possibly to rationally predict how changing any one such variable, e.g. the cross-link ratio, is likely to impact upon these properties [44-48].

\subsection{Template}

The template is central importance and it directs organization of the functional groups pendent to the functional monomers in all molecular imprinting processes. In terms of compatibility with free radical polymerization, templates should ideally be chemically inert under the polymerization conditions, thus alternative imprinting strategies may have to be sought if the template can participate in radical reactions or is for any other reason unstable under the polymerization conditions. The following are legitimate questions to ask of a template: (1) Does the template bear any polymerisable groups? (2) Does the template bear functionality that could potentially inhibit or retard a free radical polymerization? (3) Will the template be stable at moderately elevated temperatures or upon exposure to UV irradiation? The imprinting of small, organic molecules (e.g., pharmaceuticals, pesticides, amino acids and peptides, nucleotide bases, steroids, and sugars) is now well established and considered almost routine. Optically active templates have been used in most cases during optimization. In these cases the accuracy of the structure of the imprint (the cavity with binding sites) could be measured by its ability for racemic resolution, which was tested either in a batch procedure or by using the polymeric materials as chromatographic supports.

One of the many attractive features of the molecular imprinting method is that it can be applied to a diverse range of analytes, however, not all templates are directly amenable to molecular imprinting processes. Most routine MIP were using small organic molecules as template. Although specially adapted protocols have been proposed for larger organic compounds, e.g., proteins, cells, imprinting of much larger structures is still a challenge. The primary reason is the fact that larger templates are less rigid and thus do not facilitate creation of well-defined binding cavities during the imprinting process. 
Furthermore, the secondary and tertiary structure of large biomolecules such as proteins may be affected when exposed to the thermal or photolytic treatment involved in the synthesis of imprinted polymers. Rebinding is also more difficult, since large molecules such peptides and proteins do not readily penetrate the polymer network for reoccupation of binding pockets.

\subsection{Monomers}

The careful choice of functional monomer is one of the utmost importance to provide complementary interactions with the template and substrates. (Figure 2) For covalent molecular imprinting, the effects of changing the template to functional monomer ratio is not necessary because the template dictates the number of functional monomers that can be covalently attached; furthermore, the functional monomers are attached in a stoichiometric manner. For non-covalent imprinting, the optimal template /monomer ratio is achieved empirically by evaluating several polymers made with different formulations with increasing template [49]. The underlying reason for this is thought to originate with the solution complex between functional monomers and template, which is governed by Le Chatelier's principle. Applying Le Chatelier's principle to the complex formed prior to polymerization, increasing the concentration of components or binding affinity of the complex in the prepolymerization mixture would predict an increase in the pre-polymer complex. Correspondingly, there is an increase the number of final binding sites in the imprinted polymer, resulting in an increased binding or selectivity factor per gram of polymer.

From the general mechanism of formation of MIP binding sites, functional monomers are responsible for the binding interactions in the imprinted binding sites, and for non-covalent molecular imprinting protocols, are normally used in excess relative to the number of moles of template to favor the formation of template-functional monomer assemblies. It is very important to match the functionality of the template with the functionality of the functional monomer in a complementary fashion (e.g. H-bond donor with H-bond acceptor) in order to maximise complex formation and thus the imprinting effect. Higher retention and resolution was finding by the two co-monomer imprinting polymer than the single monomer imprinting polymer, which indicated an increase in the affinity of the MIP with the sample as a result of the cooperation effect of the binding sites. However, it's important to bear reactivity ratios of the monomers to ensure those copolymerisations are feasible. 
<smiles>C=CC(=O)O</smiles>

acrylic acid<smiles>C=C(C)C(=O)O</smiles>

methacrylic acid<smiles>C=C(C)C(=O)OC</smiles>

methyl methacrylic acid<smiles>C=Cc1ccc(C(=O)O)cc1</smiles>

p-vinylbenzoic acid<smiles>C=CC(CC(=O)O)C(=O)O</smiles>

itaconic acid<smiles>C=Cc1ccc(CC)cc1</smiles>

4-ethystyrene<smiles>C=Cc1ccccc1</smiles>

4-ethystyrene<smiles>C=Cc1ccncc1</smiles>

4-vinylpyridine<smiles>C=Cc1ccccn1</smiles>

2-vinylpyridine<smiles></smiles>

1-vinylimidazole<smiles>C=CC(=O)CC(C)(C)CS(=O)(=O)O</smiles>

acrylamido-2-methyl-1-propane- sulphonic acid<smiles>C=CC(N)=O</smiles>

acrylamide<smiles>C=C(C)C(N)=O</smiles>

methacrylamide

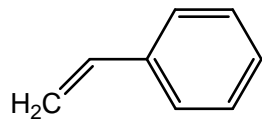

styrene

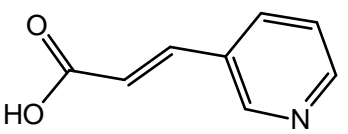

trans-3-(3-pyridyl)-acrylic acid

Figure 2. Common functional monomers used in non-covalent molecular imprinting procedures.

\subsection{Crosslinkers}

The selectivity is greatly influenced by the kind and amount of cross-linking agent used in the synthesis of the imprinted polymer. The careful choice of functional monomer is another importance choice to provide complementary interactions with the template and substrates (Figure 3). In an imprinted polymer, the cross-linker fulfils three major functions: First of all, the cross-linker is important in controlling the morphology of the polymer matrix, whether it is gel-type, macroporous or a microgel powder. Secondly, it serves to stabilize the imprinted binding site. Finally, it imparts mechanical stability to the polymer matrix. From a polymerization point of view, high cross-link ratios are generally preferred in order to access permanently porous (macroporous) materials and in order to be able to generate materials with adequate mechanical stability. So the amount of cross-linker should be high enough to maintain the stability of the recognition sites. These may be because the high degree of cross-linking enables the microcavities to maintain three-dimensional structure complementary in both shape and chemical functionality to that of the template after removal of the template, and thus, the functional groups are held in an optimal configuration for rebinding the template, allowing the receptor to 'recognize' the original substrate. Polymers with cross-link ratios in excess of $80 \%$ are 
often be used. Quite a number of cross-linkers compatible with molecular imprinting are known, and a few of which are capable of simultaneously complexing with the template and thus acting as functional monomers.

\subsection{Porogenic solvents}

Porogenic solvents play an important role in formation of the porous structure of MIP, which known as macroporous polymers. It is known that the nature and level of porogenic solvents determines the strength of non-covalent interactions and influences polymer morphology which, obviously, directly affects the performance of MIP. Firstly, template molecule, initiator, monomer and cross-linker have to be soluble in the porogenic solvents. Secondly, the porogenic solvents should produce large pores, in order to assure good flow-through properties of the resulting polymer. Thirdly, the porogenic solvents should be relatively low polarity, in order to reduce the interferences during complex formation between the imprint molecule and the monomer, as the latter is very important to obtain high selectivity MIP.

Porogenic solvents with low solubility phase separate early and tend to form larger pores and materials with lower surface areas. Conversely, porogenic solvents with higher solubility phase separate later in the polymerization provide materials with smaller pore size distributions and greater surface area. More specifically, use of a thermodynamically good solvent tends to lead to polymers with well developed pore structures and high specific surface areas, use of a thermodynamically poor solvent leads to polymers with poorly developed pore structures and low specific surface areas. However, the binding and selectivity in MIP is not appeared to dependent on a particular porosity.

Although the results of molecular recognition weaken with the polar of the porogenic solvents increasing, however, it is important to stress that in some cases sufficiently strong template: monomer interactions have been observed in rather polar solvents (e.g. methanol/water). Increasing the volume of porogenic solvents increases the pore volume. Besides its dual roles as a solvent and as a pore forming agent, the solvent in a non-covalent imprinting polymerization must also be judiciously chosen such that it simultaneously maximizes the likelihood of template, functional monomer complex formation. Normally, this implies that apolar, non-protic solvents, e.g. toluene, are preferred as such solvents stabilize hydrogen bonds, however if hydrophobic forces are being used to drive the complexation then water could well be the solvent of choice. 
<smiles>C=CC(=O)Nc1ccc(NC(=O)C=C)cc1</smiles>

$\mathrm{N}, \mathrm{N}^{\prime}$-1,4-phenylenediacrylamine<smiles>C=C(C)C(=O)OCCOC(=O)C(C)=O</smiles>

ethylene glycol dimethacrylate<smiles>C=Cc1ccc(C=C)cc1</smiles>
divinylbenzene<smiles>C=C(C)c1cccc(C(=C)C)c1</smiles>
1,3-diisopropenyl benzene<smiles>C=CC(=O)Nc1cccc(NC(=O)C=C)n1</smiles>
2,6-bisacryloylamidopyridine<smiles>C=C(C)C(=O)OCC(CC)(COC(=O)C(=C)C)COC(=O)C(=C)C</smiles>

trimethylpropane trimethacrylate<smiles>C=CC(=O)NCNC(=O)C=C</smiles>

$\mathrm{N}, \mathrm{N}$ '-methylenediacrylamide<smiles>C=CC(=O)Nc1cc(NC(=O)C=C)cc(C(=O)O)c1</smiles>

3,5-bis(acryloylamido)benzoic acid<smiles>C=CC(=O)NC(COC(=O)C=C)Cc1ccccc1</smiles>

N,O-bisacryloyl-phenylalaninol<smiles>C=C(C)C(=O)OCCCCOC(=O)C(=C)C</smiles>

tetramethylene dimethacrylate<smiles>C=CC(=O)N1CCN(C(=O)C=C)CC1</smiles>

1,4-diacryloyl piperazine<smiles>C=CC(=O)OCC(COC(=O)C=C)(COC(=O)C=C)COC(=O)C=C</smiles>

pentaerythritol tetraacrylate

Figure 3. Chemical structure of common cross-linkers used in non-covalent molecular imprinting. 


\subsection{Initiators}

Many chemical initiators with different chemical properties can be used as the radical source in free radical polymerization (Figure 4). Normally they are used at low levels compared to the monomer, e.g. 1 wt. $\%$, or $1 \mathrm{~mol}$. \% with respect to the total number of moles of polymerisable double bonds. The rate and mode of decomposition of an initiator to radicals can be triggered and controlled in a number of ways, including heat, light and by chemical/electrochemical means, depending upon its chemical nature. For example, the azoinitiator azobisisobutyronitrile (AIBN) can be conveniently decomposed by photolysis (UV) or thermolysis to give stabilised, carbon-centred radicals capable of initiating the growth of a number of vinyl monomers. As an illustrative example of the use of AIBN, or indeed other initiators, to polymerize vinyl monomers, AIBN can polymerize methylmethacrylate under thermal or photochemical conditions to give poly(methyl methacrylate).

Oxygen gas retards free radical polymerizations, thus in order to maximize the rates of monomer propagation, ensure good batch-to-batch reproducibility of polymerizations, removal of the dissolved oxygen from monomer solutions immediately prior to proliferation is advisable. Removal of dissolved oxygen can be achieved simply by ultrasonication or by sparging of the monomer solution by an inert gas, e.g. nitrogen or argon.<smiles>CC(C)(C#N)N=NC(C)(C)C#N</smiles>

azobisisobutyronitrile

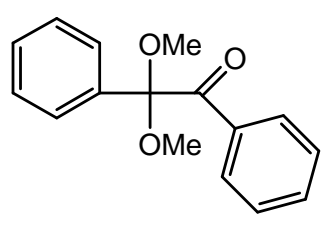

dimethylacetal of benzil

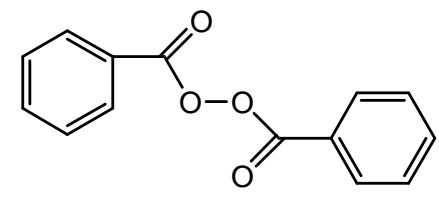

benzoylperoxide

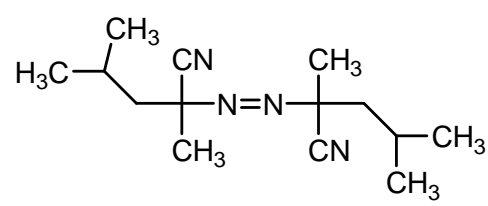

azobisdimethylvaleronitrile

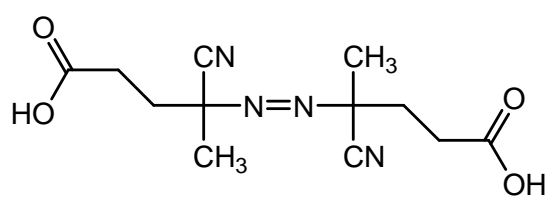

4,4'-azo(4-cyanovaleric acid)

Figure 4. Chemical structure of common initiators used in non-covalent molecular imprinting

\subsection{Polymerization condition}

Several studies have shown that polymerization of MIP at lower temperatures forms polymers with greater selectivity versus polymers made at elevated temperatures. Usually, most people using $60^{\circ} \mathrm{C}$ as the polymerization temperature. However, the initiation of the polymerization reaction was very fast and therefore hard to control at this temperature and resulted in low reproducibility of molecular imprinted polymer. Furthermore, the relatively high temperatures have a negative impact on the complex stability, which reduced the reproducibility of the monolithic stationary phases and produced high column pressure drops. Thus, the relatively low temperatures of with a prolonged reaction time 
were selected in order to yield a more reproducible polymerization. Where complexation is driven by hydrogen bonding then lower polymerization temperatures are preferred, and under such circumstances photochemically active initiators may well be preferred as these can operate efficiently at low temperature. For example, Mosbach et al. [50-51] presented a study on enantioselectivity of 1-PheNHPh imprinted polymers, one polymer being thermally polymerized at $60^{\circ} \mathrm{C}$, the other photochemically polymerized at $0^{\circ} \mathrm{C}$. The results showed that better selectivity is obtained at the lower temperature versus the identical polymers thermally polymerized. The reason for this has again been postulated on the basis of Le Chatelier's principle, which predicts that lower temperatures will drive the pre-polymer complex toward complex formation, thus increasing the number and, possibly, the quality of the binding sites formed.

\section{Preparation Methods of MIP}

\subsection{Bulk Polymerization}

Molecularly imprinted polymers can be prepared in a variety of physical forms to suit the final application desired (Table 1). The conventional method for preparing MIP is via solution polymerization followed by mechanical grinding of the resulting bulk polymer generated to give small particles and sieve the particles into the desired size ranges, which diameters usually in the micrometer range [52-53]. This method, by far the most popular, presents many attractive properties, especially to newcomers. In fact, it is fast and simple in its practical execution and it does not require particular operator skills or sophisticated instrumentation. Particle sizes $<25 \mu \mathrm{m}$ are usually used in chromatographic studies [54]. Such ground and sieved particles have been packed into conventional HPLC columns, immobilized on TLC plates, and entrapped in capillary columns using acrylamide gels or silicate matrices.

Although bulk polymerization is simple, and optimization of imprinting conditions is relatively straightforward, however, bulk polymerization method presents many drawbacks anyway. First of all, the particles obtained after the last sieving step have a highly irregular in size and shape, some interaction sites are destroyed during grinding, and thus lead to a negative impact on chromatographic performance and lower MIP loading capacity with respect to theoretical values. Moreover, the procedure of grinding and sieving is cumbersome, and it causes a substantial loss of useful polymer, that can be estimated between 50 and $75 \%$ of the initial amount of bulk material. Since a portion of polymer can only be used as packing material, this method suffered high consumption of the template molecules. Last, but not least, due to its exothermical nature, bulk polymerization cannot be scaled-up without danger of sample overheating.

Gonzalez et al. [55] systemic studied the non-covalent MIP synthesized by "bulk" polymerization using digoxin as template. These polymers were synthesized under different conditions, i.e., changing the functional monomers employed (methacrylic acid or 2-vinylpyridine), the porogenic solvents (acetonitrile or dichloromethane) used, different polymerization and extraction processes were used. The polymerization process was proceeding either under UV light or in a thermostat-controlled water bath. This produced polymers with different structural conformations and characteristics (hardness, 
porosity, stiffness, loading capacity, strength, etc.). The binding capacity, binding specificity and chemical and thermal capacities of these MIP were found to depend directly on the characteristics of their surface morphology.

Molecular imprinting of 2-aminopyridine (2-apy) in bulk polymerizations of acrylic and sol-gel based polymers has been synthesized by O’Mahony [56]. Both polymeric systems reveal varying degrees of affinity in rebinding the original template as well as a number of structural analogues. Rebinding was conducted in chloroform, acetonitrile and methanol in order to assess the role of hydrogen bonding in imprinting. The acrylic imprinted polymer retained approximately $50 \%$ of the template in rebinding studies in chloroform compared to $100 \%$ for the sol-gel. However, this higher affinity for the sol-gel was accompanied by a higher degree of non-specific binding. The acrylic polymer exhibited little discrimination between imprinted and reference polymers for 3-aminopyridine (3-apy) indicating the high selectivity of the MIP polymer for 2-apy relative to 3-apy.

Table 1. Summary of MIP prepared by different methods.

\begin{tabular}{|c|c|c|}
\hline MIP format & Benefits & Limitations \\
\hline Bulk polymerization & $\begin{array}{l}\text { Polymerization simplicity and } \\
\text { universality, } \\
\text { No require particular skills or } \\
\text { sophisticated instrumentation }\end{array}$ & $\begin{array}{l}\text { Tedious procedures of grinding, } \\
\text { sieving, and column packing, } \\
\text { Irregular particle in size and shape, } \\
\text { low performance. }\end{array}$ \\
\hline Suspension polymerization & $\begin{array}{l}\text { spherical particles, } \\
\text { Highly reproducible results, } \\
\text { Large scale possible }\end{array}$ & $\begin{array}{l}\text { Phase partitioning of complicates } \\
\text { system, } \\
\text { Water is incompatible with most } \\
\text { imprinted procedures, Specialist } \\
\text { surfactant polymers required }\end{array}$ \\
\hline $\begin{array}{l}\text { Multi-step swelling } \\
\text { polymerization }\end{array}$ & $\begin{array}{l}\text { Monodisperse beads of } \\
\text { controlled diameter, } \\
\text { Excellent particle for HPLC }\end{array}$ & $\begin{array}{l}\text { Complicated procedures and } \\
\text { reaction conditions, } \\
\text { Need for aqueous emulsions, }\end{array}$ \\
\hline Precipitation polymerization & $\begin{array}{l}\text { Imprinted microspheres, } \\
\text { Uniform size and high yields }\end{array}$ & $\begin{array}{l}\text { Large amount of template } \\
\text { High dilution factor }\end{array}$ \\
\hline Surface polymerization & $\begin{array}{l}\text { Monodisperse product, } \\
\text { Thin imprinted layers }\end{array}$ & $\begin{array}{l}\text { Complicated system, } \\
\text { Time consuming }\end{array}$ \\
\hline In-situ polymerization & $\begin{array}{l}\text { One-step, in-situ preparation, } \\
\text { Cost-efficient, good porosity }\end{array}$ & $\begin{array}{l}\text { Extensive optimization required } \\
\text { for each new template system }\end{array}$ \\
\hline
\end{tabular}

\subsection{Multi-step Swelling Polymerization}

In recent years, much effort has been dedicated to developing alternative methods to prepare imprinted stationary phases, which are superior in terms of efficiency and mass transfer properties. 
Micrometer-sized spherical imprinted polymers with narrow size distribution have been prepared through several techniques [57-59]. Uniformed spherical particles have been obtained by using multi-step swelling method [60-63]. Particles can be prepared directly in the form of spherical beads of controlled diameter. Beads synthesized in this way can be rendered magnetic through inclusion of iron oxide particles. Although particles obtained using this technique are comparatively monodisperse in size and shape and well suited for chromatographic applications, however, fairly complicated procedures and reaction conditions are required, and the aqueous suspensions used in this technique could interfere with the imprinting and thus lead to a decrease in selectivity. The requirement for aqueous emulsions can interfere with the imprinting process and the selectivity of these particles is still not completely satisfactory.

Uniformly sized MIP for d-chlorpheniramine (CP) and -brompheniramine (BP) prepared by a multi-step swelling polymerization method and evaluated using a mixture of phosphate buffer and acetonitrile as mobile phase [64]. CP and BP enantiomers were retained the most as a monovalent cation on MAA-co-EDMA polymers and a divalent cation on TFMAA-co-EDMA polymers. Ion exchange and hydrophobic interactions could mainly work for the retention and enantioseparation of $\mathrm{CP}$ and BP on both MAA-co-EDMA and TFMAA-co-EDMA polymers in hydro-organic mobile phases.

\subsection{Suspension Polymerization}

A rather simple method for the preparation of imprinted supports not requiring mechanical grinding is suspension polymerization, which yields aggregates of spherical particles, if the system is sufficiently dilute, uniformly sized microspheres. To avoid above interference in multi-step swelling method, suspension polymerization in perfluorocarbon solvents has been studied [65-66]. In two-phase systems, the use of liquid perfluorocarbons instead of water as the continuous phase might be preferred since water may have a detrimental effect on the non-covalent complex between monomers and imprint molecule. Although regular molecularly imprinted microspheres have been prepared and excellent chromatographic performance was obtained from polymer beads produced by use of these methods and selectivity was good even at high flow rates, unfortunately, the specialized perfluorocarbon solvent and fluorinated surfactant impose limits on the applicability and practicality of this method.

Bovine serum albumin-imprinted polyacrylamide gel beads were synthesized via inverse-phase seed suspension polymerization, using high-density crosslinked gel beads as core, low-density crosslinked polyacrylamide gel as imprinting shell [67]. The selectivity test showed that imprinting gel beads exhibited good recognition for template proteins, as compared to the control protein. The imprinting beads had quick adsorption rate and possessed improved regeneration property in comparison with those prepared directly via inverse-phase suspension polymerization. They consider the formation of multiple hydrogen bonds and complementary shape between the imprinting cavities and the template proteins are the two factors that lead to the imprinting effect. 
A MIP that uses a stable isotope labeled compound as the template molecule, the so-called IMIP, was developed by suspension polymerization [68]. The selectivity factors of MIP and IMIP for bisphenol A (BPA) were 4.45 and 4.43, respectively. Therefore, IMIP was found to have the same molecular recognition ability as MIP. When MI-SPE with IMIP was used and followed by LC-MS in the analysis of river water sample, the detection limit of BPA was 1 ppt with high sensitivity.

\subsection{Precipitation Polymerization}

MIP microspherical shapes with more uniform size can be obtained by the method of precipitation polymerization, which offers a higher active surface area by manipulating its compositions. As regards precipitation polymerization, this technique involves coagulation of nano-gel beads followed by ordered particle growth due to capture of oligomers from surrounding solution [69-70]. In this manner, near-monodispersed spherical beads can be prepared, and size and porosity can be fine-tuned thereby changing the polymerization conditions. This technique has been reported in MIP-based competition assays [71-72] and capillary electrochromatography [73-74], but only recently works have been published, in which it is clearly shown that precipitation polymerization can be a potentially fruitful technique for preparing chromatography-grade molecularly imprinted beads [75].

Recently, precipitation polymerization has been employed for the production of molecularly imprinted microspheres by Ye and Puoci et al [76-78]. Despite the higher yields, a large amount of template molecules is needed for the preparation process, because of the high dilution factor. The feasibility of preparing highly selective morphine imprinting polymer particles using precipitation polymerization is demonstrated by Ho et al. [79]. Since the template, morphine hydrochloride, is dilute in the polymerization solution, it is assumed that the $\mathrm{pH}$ effect can be neglected in the system. The MIP prepared by precipitation polymerization gave uniform particles, and this proved to be a feasible method for fabricating MIP. By controlling the separation point during the cross-linking polymerization process, starting with a dilute monomer solution, uniform molecular imprinted microspheres were obtained in good yield. Compared with the traditional method, these particles exhibit greater recognition binding ability in the sensing of morphine without a time-consuming process of grinding and sieving. The rebinding of MIP by the precipitation polymerization in a solution containing morphine exhibited better performance than that of NMIP.

Baggiani [80] prepare near monodispersed polystyrene beads by precipitation polymerization in acetonitrile, and these polymeric beads cheap and easy-to-make, represent a convenient alternative to the expensive chromatography-grade silica in the iniferter-mediated grafting of MIP. The chromatographic behaviour of a column packed with these imprinted beads was compared with another column packed with irregular particles obtained by grinding of a bulk pyrimethanil-imprinted polymer. These results are consistent with an influence of the polymerization method on the morphology of the resulting polymer and not on the molecular recognition properties due to the molecular imprinting process. 


\subsection{Surface Imprinting Polymerization}

Surface grafting of MIP layers onto preformed beads has been recently proposed as attractive and apparently general techniques to obtain chromatography-grade imprinted materials. In this method, thin imprinted layers have been successfully used as coatings on chromatography-grade porous silica using several techniques to restrain the radical polymerization at the surface of the beads [81-82].

An imprinted layer selective to specific molecules on the surface of widely used polymers without affecting the bulk features was prepared by Sreenivasan [83]. The methodology is simple and modified surface could be used in applications as diverse as separation, sensing, medical uses, etc. Say et al. [84] prepared phosphorotriesterase mimic surface imprinted polymeric microbeads using MAH- $\mathrm{Cu}(\mathrm{II})$ as a new metal-chelating monomer. The paraoxon hydrolytic activity results showed that hydrolytic activity of PIBs was higher than NIBs. The preparation of the polymer is simple, inexpensive and results demonstrated that the catalytic activity of microbeads has decreased by only $17 \%$ after several uses.

The surface imprinting technique utilizing water-in-oil (W/O) emulsions was applied to the preparation of a metal ion-imprinted membrane by Araki [85]. The zinc(II) ionimprinted membrane was successfully prepared by emulsion polymerization with 1,12-dodecanediol-O,O'-diphenylphosphonic acid (functional host molecule), 1-glutamic acid dioleylester ribitol (emulsion stabilizer), and divinylbenzene (polymer matrix-forming monomer). To obtain flexible and mechanically stable membranes for practical applications, the polymerization was conducted in the presence of acrylonitrile-butadiene rubber and hydrophilized poly(tetrafluoroethylene) membranes. The use of acrylonitrile-butadiene rubber and a porous solid support in the polymer matrix resulted in improved flexibility and mechanical strength of the imprinted membrane. The permeation mechanism of the metal ions was considered to be hopping of metal ions on the binding sites in the membranes.

Piacham et al. [86] prepare ultra-thin MIP films using surface initiated radical polymerization. Polymer films are directly formed on gold-coated quartz crystal resonator, which offers easy monitoring of polymer growth. With this approach its easy control the thickness of the MIP film to be below $50 \mathrm{~nm}$, where the selective recognition of target analytes can be easily detected by the underlying quartz crystal resonator. When used in a flow injection analysis system, the assembled QCM sensor generated a large frequency change $(>30 \mathrm{~Hz})$ upon encountering a small amount of analyte $(0.19 \mathrm{mM})$. The sensor had a very short response time $(<1 \mathrm{~min})$, and displayed certain chiral selectivity towards the original template, (S)-propranolol at a concentration higher than $0.38 \mathrm{mM}$ in aqueous solution.

\subsection{Monolithic Imprinted Polymerization}

Monolithic molecularly imprinted technology combined the advantage of monolithic column and molecular imprinted technology, which was prepared by a simple, one-step, in-situ, free-radical polymerization "molding', process directly within a chromatographic column without the tedious 
procedures of grinding, sieving, and column packing. Monolithic MIP is expected to improve the separation and enable direct analysis with high-speed and high performance after in-situ polymerization. Matsui et al. [87-88] first used the in-situ polymerization technique for preparation of molecularly imprinted monoliths. Template, functional monomer, cross-linker and initiator were dissolved in mixture porogenic solvents (cyclohexanol and 1-dodecanol) and the mixture was degassed and poured into a stainless steel column. After polymerization, the template and porogenic solvents were removed by exhaustive washing with methanol-acetic acid. The monolithic molecularly imprinted technology has attracted significant interest because of their ease of preparation, high reproducibility, high selectivity and sensitivity, and rapid mass transport. Furthermore, the preparation of this type of MIP is more cost-efficient, because the amount of template molecules required is much lower. Moreover, their greater porosity, and hence good permeability, and high surface area are well suited for both small molecules and large biopolymers. Monolithic molecularly imprinted stationary phases have become a rapidly expanding field in chromatographic stationary phase preparation in recent years [89].

The proportion of mixture composition and polymerization temperature defines the monolithic structure and separation characteristic without further processing. The key to successful column preparation of MIP monolith is both choice of the composition of the pre-polymerization mixture and porogenic solvents and careful timing of the polymerization reaction. In order to compare the different polymerization methods, Mayes et al. [90] prepared three types of MIP by bulk, multistep-swelling and grafting methods when some $\beta$-blockers were used as the template molecules. In that study, ground monolithic imprinted polymer was thought to be the best all-round performer for enantiomeric separations of drugs by HPLC. In-situ polymerization has similar recognition ability and possesses the advantages of a one-step preparation procedure and high yield. Matsui and Huang [91-93] prepared a set of monolithic molecularly imprinted polymers with cinchonine and amino acid derivatives as the template molecules. Separation of the corresponding enantiomers was achieved but the separation mechanism was not mentioned. In recent years, the uses of monolithic media for superior chromatographic separation in high-performance liquid chromatography and capillary electrochromatography have attracted considerable attention [94-102].

Imprinted monolithic membranes by polymerizing mixtures of methacrylic acid and dimethacrylate crosslinkers within microporous support of filtered paper is described by Kiełczynski [103]. These membranes are selectively permeable for template molecules but transport of others species is mostly limited. The measured transport stereoselectivity varied from 1.1 to 3.7 depending on the system used and the presence of cinchonine in the monomer mixture made membrane more permeable while cinchonidine reduced its permeability. Optimization of the imprinted polymer membrane in terms of the number of binding sites and their selectivity for the template enantiomer ought to be connected with optimization of membrane porosity and template interactions with functional groups. When EDMA and EDMA:MAA monolithic membranes behave in the predicable way, i.e. transport of cinchona alkaloids follows membrane templating, TEGDMA-family membranes show abnormal preference to transport cinchonidine more effectively. 


\section{Conclusion and Future Outlook}

Molecular imprinting, as a technique for the creation of artificial receptor-like binding sites with a "memory" for the shape and functional group positions of the template molecule, has become increasingly attractive in many fields of analytical chemistry in recent years. However, there are still many formidable tasks remaining to be tackled. One particular goal is to be able to prepare molecularly imprinted polymers with a homogeneous population of binding sites, similar to monoclonal antibodies. New synthesis method with more simplicity and higher selectivity is another main goal. Improved performance of polymers in aqueous systems, quantitative analysis real samples and imprinted for large biomolecules are also desirable. Molecular imprinting technology is now generating increasing industrial interest, and there is a demand for commercially relevant applications. Molecular imprinting technology not only the principles have to be demonstrated, but their applicability to the 'real world' has also to be shown in the future.

\section{Acknowledgments}

The authors gratefully appreciate the financial support by the Center for Advanced Bioseparation Technology of Inha University, Korea.

\section{References and Notes}

1. Dickey, F.H. The preparation of specific adsorbents. Proc. Natl. Acad. Sci. 1949, 35, 227-229.

2. Owens, P.K.; Karlsson, L.; Lutz, E.S.M.; Andersson, L.I. Molecular Imprinting for Bio- and Pharmaceutical Analysis. Trend. Anal. Chem. 1999, 18, 146-154.

3. Yin, J.; Yang, G.; Chen, Y. Rapid and efficient chiral separation of nateglinide and its l-enantiomer on monolithic molecularly imprinted polymers. J.Chromatogr. A 2005, 1090, 68-75.

4. Shea, K.J.; Sassaki, D.Y. On the control of microenvironment shape of functionalized network polymers prepared by template polymerization. J. Am. Chem. Soc. 1989, 111, 3442-3444.

5. Rimmer, S. synthesis of molecular imprinted polymer networks. Chromatogr. 1998, 46, 470-474.

6. Vlatakis, G.; Andersson, L.I.; Miller, R.; Mosbach, K. Drug assay using antibody mimics made by molecular imprinting. Nature 1993, 361, 645-647.

7. Dickert, F.L.; Lieberzeit, P.; Tortschanoff, M. Molecular imprints as artificial antibodies—a new generation of chemical sensors. Sens. Actuators B 2000, 65, 186-189.

8. Hirayama, K.; Sakai, Y.; Kameoka, K.; Noda, K.; Naganawa, R. Preparation of a sensor device with specific recognition sites for acetaldehyde by molecular imprinting technique, Sens. Actuat. B 2002, 86, 20-25.

9. Haupt, K.; Mosbach, K. Molecularly imprinted polymers and their use in biomimetic sensors. Chem. Rev. 2000, 100, 2495-2504.

10. Ansell, R.J.; Kriz, D.; Mosbach, K. Molecularly imprinted polymers for bioanalysis: Chromatography, binding assays and 'biomimetic sensors'. Curr. Opin. Biotechnol. 1996, 7, 89-94.

11. Kriz, O.; Ramstrom, O.; Mosbach, K. Molecular imprinting: new possibilities for sensor technology. Anal. Chem. 1997, 69, 345A-349A.

12. Chianella, I.; Lotierzo, M.; Piletsky, S.A.; et al. Rational design of a polymer specific for 
microcystin-LR. Anal. Chem., 2002, 74, 1288-1293.

13. Lavignac, N.; Allender, C.J.; Brain, K.R. Current status of molecularly imprinted polymers as alternatives to antibodies in sorbent assays. Anal. Chim. Acta 2004, 510, 139-145.

14. Ye, L.; Mosbach, K. Molecularly imprinted microspheres as antibody binding mimics. React. Funct. Polym. 2001, 48, 149-157.

15. Molinelli, A.; Weiss, R.; Mizaikoff, B. Advanced solid phase extraction using molecularly imprinted polymers for the determination of quercetin in red wine. J. Agric. Food Chem. 2002, 50, 1804-1808.

16. Weiss, R.; Molinelli, A.; Jakusch, M.; Mizaikoff, B. Molecular imprinting and solid phase extraction of flavonoid compounds. Bioseparation 2001, 10, 379-387.

17. Bereczki, A.; Tolokan, A.; Horvai, G.; Horvath, V.; Lanza, F.; Hall, A.J.; Sellergren, B. Determination of phenytoin in plasma by molecularly imprinted solid-phase extraction. $J$. Chromatogr. A 2001, 930, 31-38.

18. Mullett, W.; Lai, E. Determination of theophylline in serum by molecularly imprinted solid-phase extraction with pulsed elution. Anal. Chem. 1998, 70, 3636-3641.

19. Martin, P.D.; Jones, G.R.; Stringer, F.; Wilson, I.D. Comparison of normal and reversed-phase solid phase extraction methods for extraction of $\beta$-blockers from plasma using molecularly imprinted polymers. Analyst 2003, 128, 345-350.

20. Hwang, C.C.; Lee, W.C. Chromatographic resolution of the enantiomers of phenylpropanolamine by using molecularly imprinted polymer as the stationary phase. J. Chromatogr. B 2001, 765, 45-53.

21. Peter, S.; Schweitz, L.; Nilsson, S. molecularly imprinted polymers in capillary electrochromatography: recent developments and future trends. Electrophoresis 2003, 24, 3892-3899.

22. Liu, F.; Liu, X.; Ng, S.C.; Chan, H.S. Enantioselective molecular imprinting polymer coated QCM for the recognition of 1-tryptophan. Sensors and Actuators B 2006, 113, 234-240.

23. Xie, J.; Zhu, L.; Luo, H.; Zhou, L.; Li, C.; Xu, X. Direct extraction of specific pharmacophoric flavonoids from ginko leaves using a molecularly imprinted polymer for quercetin. J. Chromatogr. A 2001, 934, 1-11.

24. Takeda, K.; Kobayashi, T. Bisphenol A. imprinted polymer adsorbents with selective recognition and binding characteristics. Sci. Tech. Adv. Mater. 2005, 6, 165-171.

25. Wulff, G.; Sharhan, A.; Zabrocki, K. Enzyme analogue built polyers and their use for the resolution of racements. Tetrahedron Lett.1973, 14, 4329-4332.

26. Wulff, G.; Vesper, R.; Grobe, E.; Sarhan, A. Enzyme-analogue built polymers, 4) on the synthesis of polymers containing chiral cavities and their use for the resolution of racemates. Makromol. Chem. 1977, 178, 2799-2816.

27. Svenson, J.; Karlsson, J.G.; Nicholls, I.A. 1H nuclear magnetic resonance study of the molecular imprinting of ( )-nicotine: template self-association, a molecular basis for cooperative ligand binding. J. Chromatogr. A 2004, 1024, 39-44.

28. Ekberg, B.; Mosbach, K. Molecular imprinting: A technique for producing specific separation materials. Trends in Biotechnolog. 1989, 7, 92-96.

29. Michael, J.; Whitecome, M.; Rodrigue, E.; Villar, P. A new method for the introduction of 
recognition site functionality into polymers prepared by molecular imprinting: synthesis and characterization of polymeric receptors for cholesterol. J. Am. Chem. Soc. 1995, 117, 7105-7111.

30. Sellergren, B.; Kenneth, J. S. Influence of polymer morphology on the ability of imprinted network polymers to resolve enantiomers. J. Chromatogr. 1993, 635, 31-49.

31. O’Mahony, J.; Molinelli, A.; Nolan, K.; Smyth, M.R.; Mizaikoff, B. Towards the rational development of molecularly imprinted polymers: 1H NMR studies on hydrophobicity and ion-pair interactions as driving forces for selectivity. Biosens. Bioelectron. 2005, 20, 1884-1893.

32. Mayes, A.G.; Mosbach, K. Molecularly imprinted polymers: useful materials for analytical chemistry?. Trends in Anal. Chem. 1997, 16, 321-332.

33. Brüggemann, O.; Haupt, K.; Ye, L.; Yilmaz, E.; Mosbach, K. New configurations and applications of molecularly imprinted polymers. J. Chromatog. A 2000, 889, 15-24.

34. Mosbach, K. Toward the next generation of molecular imprinting with emphasis on the formation, by direct molding, of compounds with biological activity (biomimetics). Anal. Chim. Acta 2001, 435, 3-8.

35. Nicholls, I.A. Thermodynamic considerations for the design of and ligand recognition by molecularly imprinted polymers, Chem. Lett. 1995, 24, 1035-1036.

36. Nicholls, I.A.; Adbo, K.; Andersson, H.S.; Andersson, P.O.; Ankerloo, J.; Hein-Dahlstrom, J.; Jokela, P.; Karlsson, J.G..; Oloffson, L.; Rosengren, J.; Shoravi, S.; Svenson, J.; Wikman, S. Can we rationally design molecularly imprinted polymers. Anal. Chim. Acta 2001, 435, 9-18.

37. Kim, H.; Guiochon, G. Comparison of the Thermodynamic Properties of Particulate and Monolithic Columns of Molecularly Imprinted Copolymers. Anal. Chem. 2005, 77, 93-102.

38. Piletska, E V.; Romero-Guerra, M.; Guerreiro, A.R.; Karim, K.; Turner, A.P.F; Piletsky, S.A. Adaptation of the molecular imprinted polymers towards polar environment. Anal. Chim. Acta 2005, 542, 47-51.

39. Spivak, D.A. Optimization, evaluation, and characterization of molecularly imprinted polymers. Advanced Drug Delivery Reviews 2005, 57, 1779-1794.

40. Cormack, P. A.G.; Elorza, A.Z. molecularly imprinted polymers: synthesis and characteristion. $J$. Chromatog. B 2004, 804, 173-182.

41. Andersson, L.I.; Nicholls, I.A.; Mosbach, K. Molecular imprinting: the current status and future development of polymer-based recognition systems. Adv. Molec. Cell Biol. 1996, 15, 647-666.

42. Mosbach, K. Molecular imprinting. Trends in Biochemical Sciences 1994, 19, 9-14.

43. Haupt, K.; Mosbach K. Plastic antibodies: developments and applications. Tibtech November 1998, $16,468-475$.

44. Katz, A.; Davis, M.E. Investigations into the mechanisms of molecular recognition with imprinted polymers. Macromolecules 1999, 32, 4113-4121.

45. L“ubke, C.; L“ubke, M.; Whitcombe, M.J.; Vulfson, E.N. Imprinted polymers prepared with stoichiometric template-monomer complexes: efficient binding of ampicillin from aqueous solutions. Macromolecules 2000, 33, 5098-5105.

46. Turner, N.W.; Piletska, E.V.; Karim, K.; Whitcombe, M.; Malecha, M.; Magan, N.; Baggiani, C.; Piletsky, S.A. Effect of the solvent on recognition properties of molecularly imprinted polymer specific for ochratoxin A. Biosensors and Bioelectronics 2004, 20, 1060-1067.

47. Oral, E.; Peppas, N.A. Dynamic studies of molecular imprinting polymerizations. Polymer 2004, 
45, 6163-6173.

48. Liu, Z.S.; Xu, Y.L.; Yan, C.; Gao, R.Y. Mechanism of molecular recognition on molecular imprinted monolith by capillary electrochromatography. J.Chromatogr. A 2005, 1087, 20-28.

49. Kim, H.; Spivak, D.A. New insight into modeling non-covalently imprinted polymers. J. Am. Chem. Soc. 2003, 125, 11269-11275.

50. Kempe, M.; Mosbach, K. Binding studies on substrate- and enantio-selective molecularly imprinted polymers, Anal. Lett. 1991, 24, 1137-1145.

51. O’Shannessy, D.J.; Ekberg, B.; Mosbach, K. Molecular imprinting of amino acid derivatives at low temperature using photolytic homolysis of azobisnitriles. Anal. Biochem. 1989, 177, 144-149.

52. Silvestri, D.; Borrelli, C.; Giusti, P.; Cristallini, C.; Ciardelli, G. Polymeric devices containing imprinted nanospheres: a novel approach to improve recognition in water for clinical uses. Anal. Chim. Acta 2005, 542, 3-13.

53. Baggiani, C.; Anfossi, L.; Baravalle, P.; Giovannoli, C.; Tozzi, C. Selectivity features of molecularly imprinted polymers recognizing the carbamate group. Anal. Chim. Acta 2005, 531, 199-207.

54. Sellergren, B.; Shea, K.J. Origin of peak asymmetry and the effect of temperature on solute retention in enantiomer separations on imprinted chiral stationary phases. J. Chromatogr. A. 1995, 690, 29-39.

55. Gonz'alez, G.P.; Hernando, P.F.; Alegra, J.S.D. A morphological study of molecularly imprinted polymers using the scanning electron microscope. Anal. Chim. Acta 2006, 557, 179-183.

56. O’Mahony, J.; Molinellib, A.; Nolan, K.; Smyth, M.R.; Mizaikoff, B. Anatomy of a successful imprint: Analysing the recognition mechanisms of a molecularly imprinted polymer for quercetin. Biosensors and Bioelectronics 2006, 21, 1383-1392.

57. Lei, J.D.; Tong A.J. Preparation of Z-1-Phe-OH-NBD imprinted microchannel and its molecular recognition study. Spectrochimica Acta Part A 2005, 61, 1029-1033.

58. Tamayo, F.G.; Titirici, M.M.; -Esteban, A. M.; Sellergren, B. Synthesis and evaluation of new propazine-imprinted polymer formats for use as stationary phases in liquid chromatography. Anal. Chim. Acta 2005, 542, 38-46.

59. Yang, K.; Liu, Z.; Mao, M.; Zhang, X.; Zhao, C.; Nishi, N. Molecularly imprinted polyethersulfone microspheres for the binding and recognition of bisphenol A. Anal. Chim. Acta 2005, 546, 30-36.

60. Haginaka, J.; Kagawa, C. Uniformly sized molecularly imprinted polymer for d-chlorpheniramine, Evaluation of retention and molecular recognition properties in an aqueous mobile phase. $J$. Chromatogr. A. 2002, 948, 77-84

61. Hosoya, K.; Yoshizako, K.; Shirasu, Y.; Kimata, K.; Araki, T.; Tanaka, N.; haginaka, J. Molecularly imprinted uniform-size polymer-based stationary phase for high-performance liquid chromatography structural contribution of cross-linked polymer network on specific molecular recognition. J. Chromatogr. A 1996, 728, 139-147.

62. Haginaka, J.; Sakai, Y. Uniform-sized molecularly imprinted polymer material for (S)-propranolol. J. Pharm. Biomed. Anal. 2000, 22, 899-907.

63. Nakamura, M.; Ono, M.; Nakajima, T.; Ito, Y.; Aketo, T.; Haginaka, J. Uniformly sized molecularly imprinted polymer for atropine and its application to the determination of atropine and scopolamine in pharmaceutical preparations containing Scopolia extract. J. Pharmaceutical and 
Biomedical Analysis 2005, 37, 231-237.

64. Haginaka, J.; Kagawa, C. Retentivity and enantioselectivity of uniformly sized molecularly imprinted polymers for d-chlorpheniramine and -brompheniramine in hydro-organic mobile phases. J. Chromatogr. B 2004, 804, 19-24.

65. Mayes, A.G.; Mosbach, K. Molecularly Imprinted Polymer Beads: Suspension Polymerization Using a Liquid Perfluorocarbon as the Dispersing Phase. Anal. Chem. 1996, 68, 3769 - 3774.

66. Zhang, L.; Cheng, G.; Fu, C. Synthesis and characteristics of tyrosine imprinted beads via suspension polymerization. React. Func. Polym. 2003, 56, 167-173.

67. Pang, X.; Cheng, Guoxiang.; Li, R.; Lu, S.; Zhang Y. Bovine serum albumin-imprinted polyacrylamide gel beads prepared via inverse-phase seed suspension polymerization. Anal. Chim. Acta 2005, 550, 13-17.

68. Kawaguchi, M.; Hayatsu, Y.; Nakata, H.; Ishii, Y.; Ito, R.; Saito, K.; Nakazawa H. Molecularly imprinted solid phase extraction using stable isotope labeled compounds as template and liquid chromatography-mass spectrometry for trace analysis of bisphenol A in water sample. Anal. Chim. Acta 2005, 539, 83-89.

69. Li, W.H.; Stover, D.H. Monodisperse cross-linked core-shell polymer microspheres by precipitation polymerization. Macromolecules 2000, 33(12): 4354-4360.

70. Downey, J.S.; McIsaac, G.; Frank, R.S.; Stover, D.H. Poly (divinylbenzene)microspheres as an intermediate morphology between microgel, macrogel, and coagulum in cross-linking precipitation polymerization. Macromolecules 2001, 34, 4534-4541.

71. Surugiu, I.; Danielsson, B.; Ye, L.; Mosbach, K.; Haupt, K. Chemiluminescence Imaging ELISA Using an Imprinted Polymer as the Recognition Element Instead of an Antibody. Anal. Chem. 2001, 73, 487-491.

72. Ye, L.; Surugiu, I.; Haupt, K. Scintillation Proximity Assay Using Molecularly Imprinted Microspheres. Anal. Chem. 2002, 74, 959-964.

73. Sp’egel, P.; Schweitz, L.; Nilsson, S. Molecularly imprinted microparticles for capillary electrochromatography: Studies on microparticle synthesis and electrolyte composition. Electrophoresis 2001, 22, 3833-3841.

74. Boer, T.; Mol, R.; deZeeuw, R.A.; deJong, G.J.; Sherrington, D.C.; Cormack, P.A.G.; Ensing, K. Spherical molecularly imprinted polymer particles: A promising tool for molecular recognition in capillary electrokinetic separations. Electrophoresis 2002, 23, 1296-1300.

75. Li, P.; Rong, F.; Yuan, C. Morphologies and binding characteristics of molecularly imprinted polymers prepared by precipitation polymerization. Polym. Int. 2003, 52, 1799-1806.

76. Ye, L.; Cormack, P.A.; Mosbach, K. Molecular imprinting on microgel spheres. Anal. Chim. Acta. 2001, 435, 187-196.

77. Ye, L.; Weiss, R.; Mosbach, K. Synthesis and characterization of molecularly imprinted microspheres. Macromolecules 2000, 33, 8239-8245.

78. Puoci, F.; Iemma, E.; Muzzalupo, R.; Spizzirri, U.G.; Trombino, S.; Cassano, R.; Picci, N. Spherical molecularly imprinted polymers (SMIPs) via a novel precipitation polymerization in the controlled delivery of sulfasalazine. Macromol. Biosci. 2004, 4, 22-26.

79. Ho, K.C.; Yeh, W.M.; Tung, T.S.; Liao, J.Y. Amperometric detection of orphine based on poly(3,4-ethylenedioxythiophene) immobilized molecularly imprinted polymer particles prepared 
by precipitation polymerization. Anal. Chim. Acta 2005, 542, 90-96.

80. Baggiani, C.; Baravalle, P.; Anfossi, L.; Tozzi. C. Comparison of pyrimethanil-imprinted beads and bulk polymer as stationary phase by non-linear chromatography. Anal. Chim. Acta 2005, 542, 125-34.

81. Sellergren, B.; Ruckert, B.; Hall, A.J. Layer-by-Layer Grafting of Molecularly Imprinted Polymers via Iniferter Modified Supports. Adv. Mater. 2002, 14, 1204-1208.

82. Ruckert, B.; Hall, A.J.; Sellergren, B. Molecularly imprinted composite materials via inifertermodified supports. J. Mater. Chem. 2002, 12, 2275-2280.

83. Sreenivasan, K. Surface imprinted polyurethane film as a chiral discriminator. Talanta 2006, 68, 1037-1039.

84. Say, R.; Erdem, M.; Ersoz, A.; Türk, H.; Denizli, A. Biomimetic catalysis of an organophosphate by molecularly surface imprinted polymers. Applied Catalysis A: General 2005, 286, 221-225.

85. Araki, K.; Maruyama, T.; Kamiya, N.; Goto M. Metal ion-selective membrane prepared by surface molecular imprinting. J. Chromatogr. B 2005, 818, 141-145.

86. Piacham, T.; Josell, A.; Arwin, H.; Prachayasittikul, V.; Ye L. Molecularly imprinted polymer thin films on quartz crystal microbalance using a surface bound photo-radical initiator. Anal. Chim. Acta 2005, 536, 191-96.

87. Matsui, J.; Kato, Y.; Takeuchi, T.; Yokoyama, K.; Tamiya, E.; Karube, I. Molecular recognition in continuous polymer rods prepared by a molecular imprinting technique. Anal Chem. 1993, 65, 2223-2224.

88. Matsui, J.; Miyoshi, Y.; Matsui, R. Rod-type affinity media for liquid chromatography prepared by in-situ molecular imprinting. Anal. Sci. 1995, 11 (6), 1017-1019.

89. Liu, H.Y.; Row, K.H.; Yang, G.L. Monolithic Molecularly Imprinted Columns for Chromatographic Separation. Chromatogr. 2005, 61, 429-432.

90. Robert, E.F.; Christophe, C.; Richard, F.V.; Mayes, A.G. A direct comparison of the performance of ground, beaded and silica-grafted MIPs in HPLC and Turbulent Flow Chromatography applications. Biosensors and Bioelectronics 2004, 20, 1098-1105.

91. Matsui, J.; Nicholls, I.A.; Takeuchi, T. Molecular recognition in cinchona alkaloid molecular imprinted polymer rods. Anal. Chim. Acta 1998, 365, 89-93.

92. Huang, X.D.; Zou, H.F.; Mao, X.Q.; Luo, Q.Z.; Chen, X.M.; Xiao, X.Z. Preparation of molecularly imprinted chiral monolithic column and its applications for separation of diastereomers. Chin. J. Chromatogr. 2002, 20, 436-438.

93. Huang, X.D.; Zou, H.F.; Chen, X.Q.; Luo, Q.Z.; Kong, L. Molecularly imprinted monolithic stationary phases for liquid chromatographic separation of enantiomers and diastereomers. $J$. Chromatogr. A 2003, 984, 273-282.

94. Svec, F., E.C. Peters, D. Sykora and J.M.J. Frechet. Design of the monolithic polymers used in capillary electrochromatography columns. J. Chromatogr. A 2000, 887, 3-29.

95. Yan, H.Y.; Jin, L.M.; Row, K.H. Special Selectivity of Molecular Imprinted Monolithic Stationary Phase. J. Liq. Chromatogr. \& Rel. Technol. 2005, 28, 3147-3155.

96. Wulff, G. Enzyme-like catalysis by molecularly imprinted polymers. Chem. Rev. 2002, 102, 1-28.

97. Huang, X.D.; Qin, F.; Chen, X.M.; Liu, Y.Q.; Zou, H.F. Short columns with molecularly imprinted monolithic stationary phases for rapid separation of diastereomers and enantiomers. J. Chromatogr. 
B 2004, 804, 13-18.

98. Quaglia, M.; Sellergren, B.; Lorenzi, E.D. Approaches to imprinted stationary phases for affinity capillary electrochromatography. J. Chromatogr. A 2004, 1044, 53-66.

99. Yan, W.; Gao, R.; Zhang, Z.; Wang, Q.; Jiang, C.V.; Yan, C. Capillary electrochromatographic separation of ionizable compounds with a molecular imprinted monolithic cationic exchange column. J. Sep. Sci. 2003, 26, 555-561.

100. Zhang, J.; He, L.; Fu, Q. Chromatographic Features and molecular reconition mechanism of a strychnine monolithic molecularly imprinted polymer. Chromatographia 2005, 62, 319-323.

101. Li, H.; Liu, Y.; Zhang, Z.; Liao, H.; Nie, L.; Yao, S. Separation and purification of chlorogenic acid by molecularly imprinted polymer monolithic stationary phase. J. Chromatogr. A 2005, 1098, 66-74.

102. Kim, H.; Georges, G. Thermodynamic functions and intraparticle mass transfer kinetics of structural analogues of a template on molecularly imprinted polymers in liquid chromatography. $J$. Chromatogr. A 2005, 1097, 84-97.

103. Kiełczy’nski, R.; Bryjak M. Molecularly imprinted membranes for cinchona alkaloids separation. Sep. Purif. Technol. 2005, 41, 231-235.

(C) 2006 by MDPI (http://www.mdpi.org). Reproduction is permitted for noncommercial purposes. 